\title{
Análisis diferencial del desgaste laboral emocional en profesionales de atención socio-sanitaria geriátrica
}

\author{
Differential Analysis of Emotional Work Wear in \\ Geriatric Socio-health Care Professionals
}

\author{
Carolina Bringas Molleda ${ }^{1}$ \\ Javier Naveiras Fernández ${ }^{2}$ \\ Francisco Javier Rodríguez Díaz ${ }^{3}$ \\ ${ }^{1}$ Universidad de Extremadura, Departamento de Psicología y Antropología, España \\ ${ }^{2}$ Dirección General de Función Pública, Consejería de Presidencia, Principado de Asturias, España \\ ${ }^{3}$ Universidad de Oviedo, Departamento de Psicología, España. Doctorado en Educación y Sociedad, \\ Universidad Andrés Bello, Chile
}

\begin{abstract}
Resumen. El síndrome de Burnout tiene un largo recorrido histórico en el ámbito público de los profesionales dedicados al cuidado de ancianos. Objetivo. En este estudio pretendemos conocer la influencia de diversas variables sociodemográficas sobre el desgaste laboral en profesionales de residencias públicas. Método. La muestra está constituida por 136 trabajadores de ambos géneros y con edades comprendidas entre 18 y 60 años, que desempeñan labores de atención directa y gestión psicosocial. Los instrumentos aplicados han sido el Inventario de burnout de Maslach \& Jackson (1986), y el Cuestionario de Salud General (GHQ-28), de Goldberg \& Hillier (1979). Resultados. Nuestros resultados refieren la existencia de diferencias por sexo (en el cansancio emocional, así como en el burnout en general) y por tiempo de antigüedad en la profesión (concretamente en realización personal y en burnout en general). Estos resultados van a reforzar la necesidad de potenciar la resiliencia de los equipos interdisciplinares de profesionales orientados a proteger la salud de las personas trabajadoras en materia de prevención de riesgos laborales.
\end{abstract}

Palabras clave. Burnout, sexo, edad, antigüedad en la profesión, cuidado de ancianos.

Abstract. Burnout syndrome has had a long history in the public arena of professionals dedicated to the care of the elderly. Objective. In this study, we aim to understand the influence of various socio-demographic variables on work-related wear of professionals in public residences. Method. The sample consists of 136 workers of both genders, aged between 18 and 60, who carry out direct care and psychosocial management tasks. The instruments applied were the burnout inventory by Maslach \& Jackson (1986), and the General Health Questionnaire (GHQ-28) by Goldberg \& Hillier (1979). Results. Our results point to the existence of differences by sex in emotional fatigue as well as in burnout in general; and by work seniority (specifically, in personal fulfillment; and in burnout in general). These results will reinforce the need to strengthen the resilience of interdisciplinary professional teams aimed at protecting the health of working people in terms of occupational risk prevention.

Keywords. Burnout, Sex, Age, Age in the profession, Elderly care.

${ }^{1}$ Carolina Bringas Molleda. Universidad de Extremadura, Departamento de Psicología y Antropología, España. Dirección postal: Facultad de Formación del Profesorado. Avda de la Universidad. Cáceres. Extremadura. España. E-mail: cbringas@unex.es

2Javier Naveiras Fernández. Dirección General de Función Pública. Consejería de Presidencia. Principado de Asturias, España. E-mail: sarievan_ls@yahoo.es ${ }_{3}^{3}$ Francisco Javier Rodríguez Díaz. Universidad de Oviedo, Departamento de Psicología, España. Doctorado en Educación y Sociedad, Universidad Andrés Bello, Chile. E-mail: franciscojavierrodriguezdiaz@gmail.com

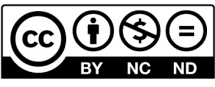

Esta obra está bajo una licencia de Creative Commons Reconocimiento-NoComercial-SinObraDerivada 4.0 Internacional. 


\section{Introducción}

La protección de la vida y la salud de los trabajadores han sido una necesaria e histórica preocupación. La promoción de la mejora de las condiciones de trabajo, a través del incremento de la seguridad y la salud de los trabajadores, se han convertido en un objetivo fundamental. El estudio científico de las emociones en el entorno laboral alcanza un enorme desarrollo en los últimos años, lo cual se circunscribe especialmente a la influencia que sobre la competencia profesional y la salud laboral pueden llegar a ejercer, repercutiendo directamente sobre la calidad del servicio ofrecido (Trujillo, Walter, Portero, Peña, \& de la Poza, 2017).

La satisfacción y la motivación del trabajador se convierten en una pieza clave en puestos vinculados a los servicios públicos, como la enseñanza, la sanidad o la atención a la tercera edad (Bullich-Marin, Basseda, Egea, Planas-Campmany, \& Juvé-Udima, 2016; Muñoz, Gómez, \& Sánchez, 2017). Dado que la protección de la salud física y psicológica de los trabajadores también repercute en aspectos como el absentismo o la productividad, más directamente ligados a los beneficios económicos de las empresas, las propias organizaciones también se han involucrado en la consecución de mejoras.

Cuando se afirma que la salud hay que defenderla, no se hace con el ánimo de demandar que se curen las lesiones o se suavicen las consecuencias del daño ya producido; se demanda la supresión de todo aquello que pudiera suponer un accidente a través de la prevención. Obviamente, desde el geriatra hasta el personal encargado de cualquier actividad laboral de los servicios públicos de atención en residencias para mayores, tiene una alta probabilidad de experimentar el síndrome de Burnout, en tanto tiene que enfrentarse y desarrollar su actividad con personas mayores.

El síndrome de Burnout es una situación de "queme" profesional, producto de una situación laboral que conlleva a nivel personal un agotamiento emocional y un sentido de despersonalización o una actitud negativa hacia el trabajo que implica un distanciamiento hacia las personas a las que prestan sus servicios (Salanova \& Llorens, 2011; Rodríguez, Rubín, Bringas, Álvarez-Fresno, \& Herrero, 2015). Ello es el resultado de la combinación de una labor de intensa responsabilidad con una disponibilidad y entrega continuamente abierta a las necesidades de los enfermos (Sánchez \& Fontalba, 2017).

A pesar de que el estrés es un fenómeno asociado a múltiples factores y que no queda circunscrito únicamente a situaciones laborales, la idea de que existe una fuerte relación entre las distintas condiciones en las que los individuos realizan su trabajo y su salud física y psicológica (Rodríguez-Díaz, Álvarez, García, Longoria, \& Noriega, 2014), hace que podamos considerar el ámbito laboral uno de los principales contextos a la hora de analizar las relaciones entre el estrés psicosocial y la salud. El estrés es un concepto complejo que implica una reacción negativa, de tipo físico o psicológico, ante una situación que resulta amenazante o que no podemos controlar, siendo en ocasiones inespecífica, y por tanto con percepción de consecuencias negativas para quien lo padece (López et al., 2012).

La salud mental en el ámbito laboral requiere una especial atención, debido a los factores que limitan el desarrollo profesional de los empleados, entre ellos: horarios de trabajo, atención a individuos enfermos que en ocasiones enfrentan crisis, el sentimiento provocado 
por la muerte, las crecientes demandas de las personas que no quedan satisfechas con los servicios recibidos, la variación constante sobre las cargas de trabajo, entre otros (Lenta \& Pérez, 2011; Montross, Meier, De Cervantes-Monteith, Vashistha, \& Irwin; 2013; Quick, Wright, Adkins, Nelson, \& Quick, 2013; Green, Savin, \& Savva, 2013).

Estos factores actúan sobre el individuo y el grupo de trabajo, provocando un malestar que puede manifestarse en fuertes cargas negativas, estrés e insatisfacción, lo que genera una deficiente calidad de la atención hacia el enfermo y en el trabajador de la salud puede producirse un estado de fatiga emocional y/o física (Gosseries et al., 2012; Haber, Palgi, Hamama-Raz, Shira, \& Ben-Ezra, 2013; Slade, 2014). Es el llamado síndrome de Burnout, que aparece con mayor frecuencia en los profesionales de las organizaciones de servicios que trabajan en contacto directo con clientes o usuarios. El padecimiento de este cansancio emocional trae consigo una deshumanización defensiva, que se manifiesta en conductas despersonalizadas, es decir, actuaciones frías y distantes con las personas que reclaman constantemente nuestra ayuda (Bringas, Fernández, Álvarez-Fresno, Martínez, \& Rodríguez-Díaz, 2015; Rodríguez et al., 2015).

Se debe resaltar que los profesionales cuando experimentan su retención de capacidades se debilitan y se transforman en seres vulnerables, especialmente para enfrentarse a situaciones difíciles; se conforma una organización "tóxica" (Hamaideh, 2014; Kubicek, Korunka, \& Ulferts, 2013), una organización de baja producción, elevado coste añadido y baja calidad de servicio, lo que tiene un efecto negativo en los usuarios. Esto último es de especial interés en una sociedad post-moderna con protagonismo de los servicios prestados en el sector terciario de los geriátricos (Butler, Brennan-Ing, Wardamsky, \& Ashley, 2014; Knopp-Sihota, Squires, Niehaus, Norton, \& Estarbrooks, 2015; Reyes \& González-Celis, 2016), que se han convertido en una ocupación imprescindible en la asistencia social y geriátrica.

La relevancia que adquiere la relación trabajador-usuario, como un factor de riesgo laboral, se concreta con las repercusiones de salud física y psíquica en el trabajador (Cabanach, Souto-Gestal, \& Franco, 2016). La tendencia en los últimos años es vincular el burnout a las variables propiciatorias para un enfoque activo y propositivo de la salud en el trabajo. En nuestro caso se constata el fracaso y la miseria en un enfrenamiento cotidiano con el sufrimiento, pobreza, crueldad, dolor, muerte, así como una preocupación por el aumento de demandas legales por una mala práctica. Una actitud analítica y empírica que se etiqueta como "modelo salutogénico laboral", donde la realidad personal y laboral es una variable dinámica y transaccional (Gil-Monte, Carretero, \& Roldán, 2005; Naveiras, 2016). Igualmente, la salud es un derecho de todos y, en esa medida, los profesionales deben estar disponibles para atender todas las necesidades que surjan como consecuencia e integrar los polos de enfermedad y bienestar de la persona.

Este enfoque supone una ventaja para el desarrollo de la actividad laboral, puesto que una organización salutogénica implica una reducción de costes (Olabarría \& Mansilla, 2007; Idris, Dollard, \& Yulita, 2014; Espinoza-Díaz, Tous-Pallarès, \& VigilColet, 2015; LeCheminant, Merrill, \& Masterson, 2015) a nivel personal (insomnio, ansiedad, depresión, adicciones, entre otros), organizacional (accidentes en el trabajo, bajas laborales, ausentismo, presentismo, entre otros), social (costes a seguridad social, 
conflicto familiar, entre otros) y comunitario (gasto sanitario en atención médica, servicios sociales, entre otros).

El agotamiento emocional pondera, como variable condicionante del burnout, a la hora de desencadenar reacciones psicosomáticas y afectaciones sobre la salud percibida; por su parte, Bernaldo-De-Quirós, Piccini, Gómez, \& Cerdeira (2015) o Le Borgne, Boudoukha, Garnier, Jeoffrion, \& Capponi (2015) han vinculado aspectos de salud y burnout con frecuencia a agresiones como riesgo de accidente psicosocial en entornos laborales. Los servicios de salud, a su vez, han encontrado relaciones entre el cansancio emocional de los trabajadores y la probabilidad de sufrir determinados actos agresivos por parte de los usuarios a los que presentan sus servicios, aunque no necesariamente violencia física (Llor-Esteban, Sánchez, Ruiz, \& Jiménez, 2017).

Gil-Monte et al. (2005), introducen la culpa en el burnout, la cual ofrece un nuevo perfil: las actitudes y conductas de indolencia como estrategia, que deterioran la calidad del servicio y dan lugar a quejas acerca del trato, frente los profesionistas con remordimientos por tratar de manera negativa e impersonal a los usuarios, en cuyo caso el burnout es más destructivo para ellos, porque manifiesta mayores problemas psicosomáticos y patologías como ansiedad y depresión (Gil-Monte, García-Juesas, \& Hernández, 2008).

Concretamente, el estudio que aquí se presenta tiene como objetivo general el detectar la naturaleza y consecuencias del servicio prestado por el personal en los Establecimientos Residenciales para Ancianos (ERA), con la finalidad de mostrar y explorar el síndrome de Burnout en estos profesionales y examinar la incidencia de variables diferenciales (género, edad y antigüedad laboral) en el desarrollo de burnout en este grupo profesional, con la intención finalista de favorecer el incrementar la calidad de vida en el trabajo y la calidad del servicio público ofrecido.

\section{Método}

\section{Participantes}

Para el estudio, de corte transversal, se procedió a una selección muestral por conveniencia, conformada por 136 trabajadores de servicios públicos, con edades comprendidas entre 18 y 60 años, procedentes del organismo autónomo ERA (Establecimientos Residenciales para Ancianos de la Comunidad Autónoma del Principado de Asturias). De la totalidad de la muestra, la población femenina la conforman las 114 personas $(83.8 \%)$, siendo varones $(n=22)$ los restantes. Asimismo, el 68.4\% ( $n=93)$ llevaban más de 10 años trabajando en la profesión. Poseían un título universitario un total de 63 trabajadores $(46.3 \%)$, mientras que 48 (35.3\%) tienen estudios de FP (Formación Profesional), y 22 (16.2\%), estudios secundarios; solo 3 personas $(2.2 \%)$ no han pasado de los estudios primarios. La categoría laboral ha estado ajustada en su mayoría por Personal de Tratamiento y Laboral $(n=127$; 93.4\%); el resto es personal de Administración y Servicios $(n=5 ; 3.7 \%)$ y pertenecientes al equipo directivo $(n=4 ; 2.9 \%)$.

\section{Instrumentos y variables}

Se administra, en primer lugar, un cuestionario ad hoc con el objetivo de recoger los datos socio-demográficos de los participantes: edad, género, nivel de estudios, centro 
de trabajo, categoría laboral, nivel económico mensual y antigüedad en el ámbito laboral. Posteriormente, se aplica el Inventario de burnout de Maslach \& Jackson (1986), adaptado por Seisdedos (1997), para evaluar el desgaste laboral a través de las sentencias a 22 ítems relacionados con la actividad profesional. Las respuestas, por medio de un formato tipo Likert, abarcan desde 0 (Nunca) a 6 (Todos los días) para categorizarse en tres factores: cansancio emocional ("me siento emocionalmente agotado por mi trabajo"), despersonalización ("creo que trato a algunos presos como si fuese objetos impersonales") y realización personal ("fácilmente comprendo cómo se sienten los presos"). La consistencia interna en el estudio original para cada uno de los factores fue: cansancio emocional (.90), despersonalización (.79) y realización personal (.71).

\section{Procedimiento}

Se contactó, en un primer momento de la investigación, a la dirección de los Establecimientos Residenciales del ERA a través de una solicitud motivada dirigida a su dirección, la cual fue enviada a la correspondiente comisión ética de la institución. Igualmente, la solicitud exponía nuestro interés y los objetivos que perseguimos una vez superadas las exigencias de la comisión ética de la Facultad de Psicología; a la vez, se asumió el compromiso de realizar un volcado de información hacia el mismo una vez finalizado el proceso. Además, se realizó un contacto telefónico con la Gerencia del organismo autónomo "ERA" (Establecimientos Residenciales para Ancianos de Asturias), para aclarar cualquier clase de duda acerca del proceso y, en virtud de este, se esperó a recibir su autorización escrita para comenzar el estudio. Ello, dio paso a la ronda de contactos directos, vía telefónica, con los diferentes centros y sus respectivos directores/ as, remitiéndoles el material de investigación y ofreciendo el mantenimiento de contacto directo para cualquier tipo dudas, aclaraciones y notificaciones. En esta dinámica, cada centro ha aplicado los mecanismos oportunos para garantizar el cubrirlo individualmente, se mantuvo el anonimato y la confidencialidad, a través de la entrega de las escalas en sobres cerrados, siendo estos recogidos por el primer autor de este estudio.

\section{Análisis de datos}

Se han categorizado las variables edad y antigüedad en la profesión en tres y dos grupos, respectivamente. Con respecto a la edad, una primera clasificación corresponde a los trabajadores con edades entre 18 y 30 años; en segundo lugar, de 31 a 50 años y, finalmente, con más de 50 años. En el caso de la antigüedad profesional se han identificado los que tenían un ejercicio acumulado de 10 años y los que referían una experiencia mayor en años.

Para responder a los objetivos planteados, los análisis realizados, procesados mediante el paquete estadístico SPSS.22, llevan, a realizar un análisis descriptivo de los tres factores de burnout en los profesionales de nuestra población del ERA, reflejando la media y desviación de cada uno de los factores, así como de la escala general, junto con los índices de fiabilidad. También, se refleja los índices de correlaciones de estos factores. A continuación, se ha realizado el contraste de medias, para el cual se ha utilizado la $t$ de Student para muestras independientes, con el objetivo de establecer las diferencias significativas en las variables diferenciales de género, edad y antigüedad en el síndrome de Burnout, tanto de manera global como en función de cada uno de los factores (cansancio emocional, despersonalización y 
realización personal). Para el caso de la edad al tener tres niveles ha implicado la utilización del ANOVA de un factor; en esta línea, se calcula el tamaño de efecto, usando la fórmula de Cohen (1988), especificando la relevancia o magnitud de las diferencias encontradas (puntuaciones por debajo de .20 indican un tamaño de efecto despreciable; entre .20 y .49 sería pequeño; entre .50 a .79, mediano y grande a partir de .80).

\section{Resultados}

Los análisis descriptivos del síndrome de Burnout de los profesionales del ERA se presentan en la Tabla 1. Se observa que existe una alta proporción de realización personal, aunque el cansancio emocional afecta a prácticamente la mitad de los trabajadores. Asimismo, se observa que el nivel de burnout es considerable. En la misma línea, se muestran los índices de fiabilidad, tanto de los factores como de la escala general, apuntándose una menor fiabilidad en el factor despersonalización. Los análisis de correlaciones que se muestran en la Tabla 2 indican una relación estadísticamente significativa y positiva entre el cansancio emocional y la despersonalización, así como en ambos casos con el burnout. En la misma línea, la correlación es significativa, pero negativa entre la despersonalización y la realización personal (ver Tabla 2).

Tabla 1

Análisis descriptivos del sindrome de Burnout ( $N=136$ )

\begin{tabular}{lccc}
\hline \multicolumn{1}{c}{ Factores } & $M$ & $D T$ & $\alpha$ \\
\hline Cansancio emocional & 25.81 & 10.39 & .75 \\
Despersonalización & 8.11 & 5.77 & .51 \\
Realización personal & 34.23 & 7.77 & .65 \\
Burnout & 47.69 & 17.41 & .76 \\
\hline
\end{tabular}

Tabla 2

Análisis de correlaciones de los factores de burnout

\begin{tabular}{lcccc}
\hline & 1 & 2 & 3 & 4 \\
\hline 1.Cansancio emocional & - & & & \\
2. Despersonalización & $.451^{*}$ & & & \\
3. Realización personal & -.066 & $-.404^{*}$ & & - \\
4. Burnout & $.776^{*}$ & $.782^{*}$ & $-.620^{*}$ & - \\
\hline${ }^{*}<.0001$ & & &
\end{tabular}


En relación con las diferencias por género, nuestros datos nos señalan que al referirnos a los elementos de queme laboral, el cansancio emocional es significativamente mayor entre las trabajadoras, observando en este punto un tamaño de efecto mediano. A nivel global, el burnout es estadísticamente mayor en las mujeres, habiendo un tamaño de efecto mediano. No se reflejan diferencias en el factor despersonalización y realización personal (ver Tabla 3).

Cuando apuntamos a la edad, se ha podido observar que no existen diferencias estadísticamente significativas en ninguno de los componentes de burnout en este ámbito laboral, y para ninguno de los agrupamientos de edad. Únicamente, se aprecia un ligero aumento del nivel de burnout en los mayores de 50 años, pero no significativo, es decir, el valor de $p$ no es inferior a .05 (ver Tabla 4).

El tiempo de antigüedad en el desempeño de la profesión ha sido también una variable objeto de nuestro estudio. Tal como apunta la Tabla 5, la realización personal se da significativamente en una proporción mayor entre quienes mayor experiencia profesional desempeñan. Con respecto al síndrome de Burnout, el queme es mayor en aquellos trabajadores que no pasan de 10 años de antigüedad en su profesión (ver Tabla 5).

Tabla 3

Prueba de medias independientes para los factores de queme laboral según el género y tamaño de efecto

\begin{tabular}{|c|c|c|c|c|c|c|c|}
\hline \multirow[t]{2}{*}{ Factores } & \multicolumn{2}{|c|}{ Hombres } & \multicolumn{2}{|c|}{ Mujeres } & \multirow[t]{2}{*}{$t$} & \multirow[t]{2}{*}{$p$} & \multirow[t]{2}{*}{ T. E. } \\
\hline & $M$ & $D T$ & $M$ & $D T$ & & & \\
\hline Cansancio emocional & 21.09 & 8.26 & 26.72 & 10.54 & -2.36 & .019 & .54 \\
\hline Despersonalización & 6.81 & 5.70 & 8.36 & 5.78 & -1.15 & .25 & .26 \\
\hline Realización personal & 35.50 & 7.84 & 33.99 & 7.77 & .83 & .40 & .19 \\
\hline Burnout & 40.40 & 16.66 & 49.10 & 17.26 & -2.17 & .031 & .50 \\
\hline
\end{tabular}

Nota. T.E. $=$ Tamaño del efecto.

${ }^{a}$ El tamaño del efecto se calculó utilizando la fórmula de Cohen (1988).

Tabla 4

Prueba de ANOVA de un factor para los factores de queme laboral según la edad

\begin{tabular}{|c|c|c|c|c|c|c|c|c|}
\hline \multirow[t]{2}{*}{ Factores } & \multicolumn{2}{|c|}{$18-30$} & \multicolumn{2}{|c|}{$31-50$} & \multicolumn{2}{|c|}{$>50$} & \multirow[t]{2}{*}{$F$} & \multirow[t]{2}{*}{$p$} \\
\hline & $M$ & DT & M & DT & M & DT & & \\
\hline Cansancio emocional & 28.25 & 12.76 & 25.06 & 11.02 & 27.04 & 8.92 & .645 & .52 \\
\hline Despersonalización & 4.25 & 3.77 & 8.58 & 6.19 & 7.55 & 4.92 & 1.40 & .24 \\
\hline Realización personal & 36.50 & 4.20 & 33.96 & 8.13 & 34.55 & 7.37 & .257 & .77 \\
\hline Burnout & 44.00 & 10.67 & 47.68 & 18.71 & 48.04 & 15.36 & .098 & .90 \\
\hline
\end{tabular}


Tabla 5

Prueba de medias independientes para los factores de queme laboral según el tiempo de antigüedad en la profesión y tamaño de efecto

\begin{tabular}{|c|c|c|c|c|c|c|c|}
\hline \multirow{2}{*}{ Factores } & \multicolumn{2}{|c|}{$\begin{array}{l}\text { Hasta } 10 \text { de } \\
\text { antigüedad }\end{array}$} & \multicolumn{2}{|c|}{$\begin{array}{l}\text { Más de } 10 \text { de } \\
\text { antigüedad }\end{array}$} & \multirow{2}{*}{$t$} & \multirow{2}{*}{$p$} & \multirow{2}{*}{ T. E. ${ }^{\mathrm{a}}$} \\
\hline & $M$ & $D T$ & $M$ & $D T$ & & & \\
\hline Cansancio emocional & 26.46 & 11.87 & 25.51 & 9.69 & .49 & .62 & .091 \\
\hline Despersonalización & 9.34 & 5.44 & 7.54 & 5.86 & 1.70 & .091 & .31 \\
\hline Realización personal & 31.51 & 7.61 & 35.49 & 7.56 & -2.84 & .005 & .51 \\
\hline Burnout & 52.30 & 16.45 & 45.56 & 17.51 & 2.12 & .036 & .38 \\
\hline
\end{tabular}

Nota. T.E. = Tamaño del efecto.

${ }^{a}$ El tamaño del efecto se calculó utilizando la fórmula de Cohen (1988).

\section{Discusión}

El balance entre demanda emocional que supone la humanización inter-contextual/multisistémica y los recursos emocionales personales (con algunos efectos conocidos tales como la "doble presencia") será quien incline la balanza en un sentido o el otro de la dimensión salud. Si consideramos que el factor de cansancio emocional es el que mejor define y condiciona el queme profesional, puede concluirse que cuantificar estas cuestiones son un reto esencial para fundamentar una mejor salud laboral y una calidad profesional de mayor excelencia. Pero dichas situaciones estáticas requieren análisis dinámicos y contextualizados.

Se reivindica los análisis integrales, sistémicos y transaccionales. Por ello, en el análisis del burnout resulta esencial desarrollar estudios globales de realidades idiosincrásicas. Habremos de definir dichas realidades con base en una serie de factores "predisponentes" versus "protectores" y ante una realidad organizacional concreta, donde diversos factores pueden tornar su dinámica como "salutogénica" o "toxigénica". Pronosticando que el factor de despersonalización actúe como un "factor reactivo de autoprotección individual" tóxico, ante el agotamiento emocional; siendo la realización profesional un "factor proactivo de protección colectiva” que actúa potenciando la resiliencia ante el agotamiento individual, salutogénico, por tanto, y que está íntimamente condicionado por el entorno emocional de los equipos de trabajo.

Los resultados permiten constatar una realidad laboral con unos valores de cansancio emocional y burnout mayores para el colectivo de personas trabajadoras pertenecientes al colectivo femenino, dentro del sector terciario público asociado al cuidado de ancianos. Esta situación podría estar mostrando un peor estado de salud en dichos colectivos profesionales, siendo ella ya resaltada previamente por algunos autores (Bachner, Yosef-Sela, \& Carmel, 2014; Ruglass \& Kendall-Tackett, 2015), contraviniendo el escenario planteado por Slade (2014). De entrada, se podría estimar una causalidad inherente a las propias condiciones laborales con repercusión sobre la propia intervención profesional sanitario-asistencial. Cuestión que no debe descuidar en su análisis el conocido efecto de la "doble presencia" (Flores, Vega, Del Río, \& Zavala, 2014). Sin embargo, esta situación podría cuestionarse con el análisis de los dos siguientes puntos: la edad y la antigüedad profesional. 
La edad de los profesionales no parece suponer una variable diferencial relevante en cuanto a las características fundamentales del burnout. Únicamente, hemos comprobado que el desgaste profesional, en su globalidad, parece afectar ligeramente a los trabajadores de mayor edad; esto es, de 50 años en adelante. Este dato resultaría contradictorio si tenemos en cuenta que la edad cronológica podría ir unida a una mayor experiencia profesional, debiendo implicar un mayor agotamiento emocional y, por ende, conllevaría además una probabilidad mayor de despersonalización a lo largo de su trayectoria laboral (Rodríguez-Díaz et al., 2014). Sorprendentemente, nuestros datos no ofrecen resultados significativos respecto a ello. Por el contrario, llama la atención que la experiencia profesional facilita el acceso a la realización profesional como posible factor de protección, pues el hecho de superar los 10 años de desempeño profesional incrementa las tasas brutas sobre este factor.

Además, el burnout como factor global afecta mayormente a aquellos trabajadores con una experiencia laboral de no más de 10 años. Cuestión por la cual se hace patente la necesidad de implementar programas de prevención/protección ante este fenómeno psicosocial en organizaciones cuyas plantillas de profesionales que, aún sin superar cierta antigüedad, requieren intervenciones que minimicen su desarrollo y, por tanto, la probabilidad de una deficiente práctica en su desarrollo laboral (Butler et al., 2014; Knopp-Sihota et al., 2015).

Evidentemente, esta acción debería darse de un modo proactivo al accidente laboral y adaptado al riesgo psicosocial. Conforme al modelo expuesto en nuestro planteamiento como de prevención primaria e intervención sobre el equipo, a diferencia de Reuben et al. (2013), preferimos nombrar "interdisciplinar", en tanto se busca potenciar el repertorio de resiliencia de un modo sinérgico (Olabarría, \& Mansilla, 2007; Fernandes et al., 2013; Woodhead, Northrop, \& Edelstein, 2016).

En esta línea de razonamiento, entendemos la realización profesional como un factor de protección ante el fenómeno del queme profesional que ha de ser analizado específicamente, potenciado en los diferentes programas específicos de intervención (a ser posible) primaria. Todo ello deberá enfocarse a la necesidad de implementar dinámicas transaccionales que canalicen todo conflicto colectivo sociocognitivo-emocional hacia la experimentación interdisciplinar de emociones positivas, como valor cultural propio de organizaciones postmodernas reflexivas y autónomas, que minimizan las sutiles percepciones de amenaza individual sobre la naturaleza manifiesta de sus demandas laborales como equipo. Una de las técnicas más conocidas es la aplicación de programas de "empoderamiento horizontal", como la formación de líderes transformadores o la tutorización/entrenamiento integral (también llamado coaching por los anglosajones) (Naveiras, 2016).

Por otra parte, a nivel de política global de recursos humanos, no resulta extraño que la paridad de género en las plantillas de profesionales actúe como factor de protección psicosocial y, por tanto, como elemento estructural en la prevención de riesgos profesionales. En nuestro estudio podemos observar una situación relativamente contradictoria, donde el colectivo femenino sufre un mayor riesgo laboral por cansancio emocional a medida que avanza su edad, pero también unos niveles de burnout que disminuyen con la antigüedad profesional. La realidad socio-cultural de nuestra muestra condiciona un rol familiar, donde la mujer asume una mayor carga emocional de manera cronificada, que ha 
de compatibilizar con la laboral. Nuestros datos pueden sostener que la "doble presencia" es un factor predisponente, precipitante o facilitador del burnout que puede ser modulada mediante la resiliencia desarrollada a través de la antigüedad profesional.

Una experiencia en el ámbito laboral, que al ser contextualizada, empodera horizontalmente y potencia competencias de afrontamiento de los equipos de trabajo. ¿Estamos vislumbrando que, a mayor consolidación de puesto, los colectivos desarrollan una mayor capacidad de afrontamiento emocional por otros factores que son implícitos? Aún podría decirse más, seguramente el compromiso profesional derivado de la consolidación de puestos y la paridad de género en las plantillas de los centros de trabajo, habrán de ser algunos de esos macro-factores.

Evidentemente, al margen de estas cuestiones estructurales, existe una realidad fenomenológica y dinámica donde se dan efectos tóxicos sobre las emociones individuales como la despersonalización, entendida como una estrategia individual de autoprotección ante el estrés y el agotamiento emocional. Una estrategia sutil que muy probablemente facilite la violencia interna en las organizaciones por parte de los propios trabajadores. Además, constata la necesidad de implementar programas psicosociales idiosincrásicos y proactivos de salud laboral que mejoren los valores de realización profesional, entendido este como un factor protector que actúa desde el nivel colectivo hacia el individual. Es en esta tesitura donde nos preguntamos si no sería conveniente establecer indicadores de "diestralidad" en las organizaciones de servicios públicos, como elemento de verdadera calidad y excelencia profesional.

Evidentemente, estas pinceladas técnicas, que aquí tratamos de trasladar, requieren de una política de recursos humanos especializada y cualificada a nivel técnico; así como realmente concienciada con la cultura preventiva, como único medio para alcanzar la excelencia en los servicios públicos. Dicho enfoque implicaría comenzar a abordar ambas cuestiones bajo unos parámetros cuantitativos y cualitativos formulados en términos positivos y propositivos como "diestralidad profesional" en las experiencias laborales, por contra de la tan conocida "siniestralidad laboral". Dichos indicadores habrían de vincular las experiencias de éxito y calidad profesional de la organización con la contingencia profesional a nivel emocional de los equipos de trabajo.

Nuestro estudio no está exento de limitaciones que podrían tenerse en cuenta a la hora de aportar datos más precisos sobre el burnout, tales como el tamaño de la muestra. Sin embargo, por nuestra realidad y nuestros resultados queremos destacar que sería necesario, junto con la experiencia profesional, tener en cuenta el rol laboral que desempeñan cada uno de los trabajadores de este ámbito residencial. Asimismo, postular la importancia de la existencia de otra variable no menos relevante en el ámbito de las organizaciones: el clima emocional que se da entre los trabajadores de estos centros asistenciales. Conocer su papel individual en la posible formación de diferentes síntomas saludables, así como en su interacción con los distintos componentes del burnout. No siendo de menor importancia el establecimiento de relaciones entre sus distintos factores con las variables de salud, satisfacción profesional o compromiso laboral. En esta línea continuaremos los análisis de nuestra investigación.

Actualidades en Psicología, 34(128), 2020, 143-156 


\section{Referencias}

Bachner, G., Yosef-Sela, N., \& Carmel, S. (2014). Open Communications with terminally ill cancer patients about illness and death. Cancer Nursing, 37(1), 50-58. doi: 10.1097/ NCC.0b013e31827b5c7a

Bernaldo-De-Quirós, M., Piccini, A., Gómez, M., \& Cerdeira, J. (2015). Psychological consequences of aggression in pre-hospital emergency care: Cross sectional Survey. International Journal of Nursing Studies, 52(1), 260-270. doi: 10.1016/j. ijnurstu.2014.05.011.

Bringas, C., Fernández, A., Álvarez-Fresno, E., Martínez, A., \& Rodríguez-Díaz, F. J. (2015). Influencia del síndrome de burnout en la salud de los trabajadores penitenciarios. Revista Española de Sanidad Penitenciaria, 17, 37-46. doi: http://dx.doi.org/10.4321/ S1575-06202015000300002

Bullich-Marín, I., Basseda, R. M., Egea, P. T., Planas-Campmany, C., \& Juvé-Udina, M. E. (2016). Evaluación del ambiente de trabajo de las enfermeras en las unidades sociosanitarias de atención intermedia de Cataluña. Revista Española de Geriatría y Gerontología, 51(6), 342-348. doi: http://dx.doi.org/10.1016/j.regg.2015.10.009

Butler, S., Brennan-Ing, M.,Wardamsky, S., \& Ashley, A. (2014). Determinants of longer job tenure among home care aides: What makes some stay on the job while others leave?. Journal of Applied Gerontology, 33(2), 164-188. doi: https://doi. org $/ 10.1177 / 0733464813495958$

Cabanach, R., Souto-Gestal, A., \& Franco, V. (2016). Escala de Estresores Académicos para la evaluación de los estresores académicos en estudiantes universitarios. Revista Iberoamericana de Psicología y Salud, 7, 41-50. doi: http://dx.doi. org/10.1016/j.rips.2016.05.001

Cohen, J. (1988). Statistical Power Analysis for the Behavioral Sciences (2nd ed.). Hillsdale, NJ: Lawrence Erlbaum Associates, Publishers.

Espinoza-Díaz, I. M., Tous-Pallarès, J., \& Vigil-Colet, A. (2015). Efecto del clima psicosocial del grupo y de la personalidad en el síndrome de quemado en el trabajo de los docentes. Anales de Psicología, 31(2), 651-657. doi: http://dx.doi.org/10.6018/ analesps.31.2.174371

Fernandes, R., Osarch, S., Bell, C. L., Flynn, B. W., Nguyen, L. V., Abad, M.J., ... Masaki, K.H. (2013). Aging and caregiving in the Republic of Palau. Journal of CrossCultural Gerontology, 28(2), 167-180. doi: 10.1007/s10823-013-9192-7

Flores, D., Vega, V., Del Río, C., \& Zavala, D. (2014). Ocuparse del bienestar de los profesionales de la salud: Un desafío pendiente. Revista chilena de terapia ocupacional, 14(1), 33-44. doi: 10.5354/0719-5346.2014.32386

Gil-Monte, P. R., Carretero, N., \& Roldán, M. D. (2005). Algunos procesos psico-sociales sobre el síndrome de quemarse por el trabajo (burnout) en profesionales de enfermería. Ansiedad y Estrés, 11(2-3), 281-291. 
Gil-Monte, P. R., García-Juesas, J. A., \& Hernández, M. C. (2008). Influencia de la Sobrecarga Laboral y la Autoeficacia sobre el Síndrome de Quemarse por el Trabajo (burnout) en Profesionales de Enfermería. Interamerican Journal of Psychology, 42(1), 113-115.

Green, L. V., Savin, S., \& Savva, N. (2013). Nursevendor problem: Personnel staffing in the presence of endogenous absenteeism. Management Science, 59(10), 2237-2256. doi: $10.1287 / \mathrm{mnsc} .2013 .1713$

Goldberg, D.P., \& Hillier, V.F. (1979). A scaled version of the General Health Questionnaire. Psychological Medicine, 9, 139-145. doi: 10.1017/s0033291700021644

Gosseries, O., Demertzi, A., Ledoux, D., Bruno, M., Vanhaudenhuyse, A., Thibaut A., \& Schnakers, C. (2012). Burnout in healthcare workers managing chronic patients with disorders of consciousness. Brain Injury, 26(12), 1493-1499. doi: http:// dx.doi.org/10.3109/02699052.2012.695426

Haber, Y., Palgi, Y., Hamama-Raz, Y., Shrira, A., \& Ben-Ezra, M. (2013). Predictors of professional quality of life among physicians in a conflict setting: The role of risk and protective factors. Israel Journal of Psychiatry and Related Sciences, 50(3), 174-181.

Hamaideh, H. (2014). Moral distress and its correlatos among mental health nurses in Jordan. International Journal of Mental Health Nursing, 23(1), 33-41. doi: 10.1111/inm.12000

Idris, M. A., Dollard, M. F., \& Yulita. (2014). Psychosocial safety climate, emotional demands, burnout, and depression: A longitudinal multilevel study in the malaysian private sector. Journal of Occupational Health Psychology, 19(3), 291-302. doi: http://dx.doi.org/10.1037/a0036599

Knopp-Sihota, A., Niehaus, L., Squires, J. E., Norton, P. G., \& Estabrooks, C. A. (2015). Factors associated with rushed and missed resident care in western canadian nursing homes: A cross-sectional survey of Elath aides. Journal of Clinical Nursing, 24(19-20), 2815-25. doi: 10.1111/jocn.12887

Kubicek, B., Korunka, C., \& Ulferts, H. (2013). Acceleration in the care of older adults: New demands as predictors of employee burnout and engagement. Journal of Advanced Nursing, 69(7), 1525-1538. doi: 10.1111/jan.12011

Le Borgne, M, Boudoukha, A., Garnier, P-H, Jeoffrion, C., \& Capponi, I. (2015). Inpatients-to-caregivers violence in geriatric settings: Impact of assaults on caregivers'mental health, risk, and protective factors. Traumatology, 21(3), 259-266. doi: https://doi.org/10.1037/trm0000046

LeCheminant, J. D., Merrill, R. M., \& Masterson, T. (2015). Health behaviors and workrelated outcomes among school employees. American Journal of Health Behavior, 39(3), 345-351. doi: http://dx.doi.org/10.5993/AJHB.39.3.7

Lenta, M., \& Pérez, K. (2011). Herramientas para la evaluación de la salud de y con los trabajadores. En Zaldúa, G. (Comp.), Epistemes y Prácticas de Psicología Preventiva. Buenos Aires, Eudeba. 
López, L., Solano, A., Arias, A., Aguirre, S., Osorio, C., \& Vásquez, E.M. (2012). El Estrés laboral y los trastornos psiquiátricos en profesionales de la medicina. Revista CES Salud Pública, 3(2), 280-288.

Llor-Esteban, B., Sánchez, M., Ruiz, J.A., \& Jiménez, J.A. (2017). User violence towards nursing professionals in mental health services and emergency units. The European Journal of Psychology Applied to Legal Context, 9, 33-40. doi: http://dx.doi. org/10.1016/j.ejpal.2016.06.002

Maslach, C., \& Jackson, S. (1986). Maslach burnout inventory manual. Palo Alto, CA: Consulting Psychologist Press, Inc.

Montross, L. P., Meier, E. A., De Cervantes-Monteith, K., Vashistha, V., \& Irwin, S. A. (2013). Hospice staff perspectives on dignity therapy. Journal of Palliative Medicine, 16(9), 1118-1120. doi: http://dx.doi.org/10.1089/jpm.2013.0030

Muñoz, T., Gómez, A., \& Sánchez, B. (2017). Satisfacción laboral en los docentes de educación infantil, primaria y secundaria. Gestión de la educación, 7(1), 161-177. doi: $10.15517 /$ rge. v7i1.27578

Naveiras, J. (2016). Salud y Burnout en el sector público de los profesionales en servicios sociales. Realidades. [Tesis Doctoral no publicada]. Universidad de Oviedo, Oviedo, España.

Olabarría, B., \& Mansilla, F. (2007). Ante el Burnout: Cuidados a los equipos de salud mental. Revista de Psicopatología y Psicología Clínica, 12(1), 1-14. doi: https://doi. org/10.5944/rppc.vol.12.num.1.2007.4029

Quick, J. C., Wright, T. A., Adkins, J. A., Nelson, D. L., \& Quick, J. D. (2013). Organizational demands, risks, and protective factors. En J. C. Quick, T. A. Wright, J. A. Adkins, D. L. Nelson, \& J. D. Quick (Eds.), Preventive stress management in organizations (2nd Ed.) (pp. 27-42) Washington, DC: American Psychological Association. doi: http://dx.doi.org/10.1037/13942-002

Reuben, B., Evertson, L. C., Wenger, S., Serrano, K., Chodosh, J., Ercoli, L., \& Tan, Z.S. (2013). The University of California at Los Angeles Alzheimer's and Dementia Care program for comprehensive, coordinated, patient-centered care: Preliminary data. Journal of the American Geriatrics Society, 61(12), 2214-2218. doi: 10.1111/jgs.12562

Reyes, K., \& González-Celis, A. L. M. (2016). Efectos de una intervención cognitivo-conductual para disminuir el burnout en cuidadores de ancianos institucionalizados. Escritos de psicología, 1(9), 15-23. doi: http://dx.doi.org/10.5231/ psy.writ.2015.1709

Rodríguez-Díaz, F. J., Álvarez, E., García, F., Longoria, B., \& Noriega, M. I. (2014). El Sistema Penitenciario: Un análisis desde el ayer para el mañana. En E. García López (Ed.). Psicopatología Forense. Comportamiento Hu-mano y Tribunales de Justicia (pp. 733- 790). México: Manual Modeno

Rodríguez, F.J., Rubín, A., Bringas, C., Álvarez-Fresno, E., \& Herrero, J. (2015). Efecto del clima emocional en el desarrollo del estrés laboral del funcionario penitenciario: Un estudio predictivo. Ansiedad y Estrés, 21(2-3), 195-205. 
Ruglass, M., \& Kendall-Tackett, K. (2015). Psychology of trauma 101. New York: Springer Publishing Co.

Salanova, M., \& Llorens, S. (2011). Hacia una perspectiva psicosocial del burnout: Cuando el trabajo "nos" quema. En E. Agullo, J. L. Álvaro, A. Garrido, R. Medina e I. Schweiger (Eds.), Nuevas formas de organización del trabajo y la empleabilidad (pp. 271296). Servicio de Publicaciones de la Universidad de Oviedo.

Sánchez, J. \& Fontalba, M. (2017). Consecuencias del Burnout de cuidadores de pacientes con demencia. European Journal of Health Research: (EJHR), 3(2), 119-126. doi: https://doi.org/10.30552/ejhr.v3i2.61

Seisdedos, N. (1997) Inventario Burnout de Maslach, Sindrome del Quemado por Estrés Laboral Asistencia. Madrid: TEA.

Slade, R. B. (2014). Understanding the role of stress and burnout in employees at nursing facilities for the elderly. [Tesis doctoral no publicada]. Department of Psychology, Capella University, Minneapolis, Minnesota, United States.

Trujillo, M.J., Walter, G., Portero, N., \& de la Poza, A. (2017). El Síndrome Burnout desde la perspectiva sanitaria. En M.M. Molero, M. C. Pérez, J. J. Gázquez, A. B. Barragán, A. Martos y M. M. Simón (Eds.), Calidad de vida, cuidadores e intervención para la mejora de la salud (pp. 17-22). Asociación Universitaria de Educación y Psicología (ASUNIVEP).

Woodhead, E. L., Northrop, L., \& Edelstein, B. (2016). Stress, social support, and burnout among long-term care nursing staff. Journal of Applied Gerontology, 35(1), 84-105. doi: $10.1177 / 0733464814542465$ 